\title{
BIRKHOFF NORMAL FORMS IN SEMI-CLASSICAL INVERSE PROBLEMS
}

\author{
A. IAntchenko, J. Sjöstrand, And M. Zworski
}

\section{Introduction}

The purpose of this note is to apply the recent results on semi-classical trace formulæ [18], and on quantum Birkhoff normal forms for semi-classical Fourier integral operators [13] to inverse problems. We show how the classical Birkhoff normal form can be recovered from semi-classical spectral invariants. In fact the full quantum Birkhoff normal form of the quantum Hamiltonian near a closed orbit, and infinitesimally with respect to the energy can be recovered. This generalizes recent results of Guillemin [8] and Zelditch [20],[21], [22] obtained in the high energy setting (a special case of semi-classical asymptotics).

We will illustrate the results in a new setting to which they apply. Let $P$ be a semi-classical Schrödinger operator:

$$
P=-h^{2} \Delta+V(x)-E, \quad V \in \mathcal{C}^{\infty}\left(\mathbb{R}^{n+1} ; \mathbb{R}\right),
$$

with the principal symbol $p(x, \xi)=\xi^{2}+V(x)-E$. We make the following assumptions:

$$
\exists \epsilon>0, \quad p^{-1}([-\epsilon, \epsilon]) \Subset T^{*} \mathbb{R}^{n+1}, \quad d p \neq 0 \text { on } p^{-1}(0) .
$$

The first assumption guarantees that $P$ has a discrete spectrum near 0 , and the second one, that the energy surface $p^{-1}(0)$ is smooth.

The semi-classical inverse problem can be formulated as follows:

What information about the energy surface, $\xi^{2}+V(x)=E$, can be recovered from the asymptotics of the spectrum of $-h^{2} \Delta+V(x)$ in a small fixed neighbourhood of $E$, as $h \rightarrow 0$ ?

This is very natural and can be considered as a mathematical formulation of the general problem arising in spectroscopy. In concrete physical situations, the relation between the Hamiltonian and the spectrum is investigated using spectroscopic Hamiltonians which are essentially Hamiltonians in Birkhoff normal forms - see [19] for one of the first uses of Birkhoff normal forms in theoretical chemistry, and [14] for recent applications using experimental spectral data.

The energy surface, $p^{-1}(0) \subset T^{*} \mathbb{R}^{n}$ has a natural bicharactistic foliation, coming from the Hamilton vector field, $H_{p}=\sum_{j=1}^{n} \partial_{\xi_{j}} p \partial_{x_{j}}-\partial_{x_{j}} p \partial_{\xi_{j}}$. Since it describes classical dynamics, its properties are a natural target of a recovery

Received January 24, 2002. 
procedure based on quantum information, such as the spectrum. A naïve intuition suggests that the quantum bound states (corresponding to eigenvalues) should correspond to the closed orbits of the classical dynamics. The simplest symplectic invariants associated to such closed orbits, $\gamma$, are the actions,

$$
S_{\gamma}=\int_{\gamma} \xi d x
$$

the eigenvalues of the linear Poincare map, $P_{\gamma}$, and the Maslov index, $\nu_{\gamma} \in \mathbb{Z}_{4}$. We recall that $P_{\gamma}$ is the differential of the (non-linear) Poincaré map, $\kappa_{\gamma}$, defined as follows: let $U$ be a hypersurface in $p^{-1}(0)$, transversal to $\gamma$, locally defined near some point on $\gamma$. Then $U$ is a symplectic manifold. The symplectic map $\kappa_{\gamma}: U \rightarrow U$ is defined as the first return map:

$$
\kappa_{\gamma}(m)=\exp \left(T(m) H_{p}\right)(m) \in U, \quad \exp \left(t H_{p}\right)(m) \notin U, 0<t<T(m),
$$

where, strictly speaking, the definition may require replacing $U$ by a larger neighbourhood as the range of $\kappa_{\gamma}$.

The relation between the spectrum and closed orbits is provided by trace formulæ: the Gutzwiller and Balian-Bloch trace formulæ in physics and their mathematical precursor, the Selberg trace formula, and successors, the DuistermaatGuillemin and the Guillemin-Melrose formulæ (see [18] and references given there). Gutzwiller's formula states that if $\operatorname{det}\left(I-P_{\gamma}^{k}\right) \neq 0$, for $0 \neq k T_{\gamma} \in \operatorname{supp} g$, then for $g, \chi \in \mathcal{C}_{\mathrm{c}}^{\infty}(\mathbb{R}), \chi \equiv 1$ near $0, g \equiv 0$ near 0 ,

$\operatorname{tr} \hat{g}\left(\frac{P}{h}\right) \chi(P) \sim \sum_{\gamma} \sum_{0 \neq k=-\infty}^{\infty} \frac{e^{i k S_{\gamma} / h+i \nu_{\gamma, k} \frac{\pi}{2}} T_{\gamma}}{\left|\operatorname{det}\left(P_{\gamma}^{k}-I\right)\right|^{\frac{1}{2}}} \sum_{j=0}^{\infty} a_{j, \gamma, k}(g) h^{j}, a_{0, \gamma, k}=g\left(k T_{\gamma}\right)$.

where $T_{\gamma}$ is the period of $\gamma$, and the $\gamma$ summation is over all simple closed orbits of $H_{p}$ on $p^{-1}(0)$. The Maslov index, $\nu_{\gamma, k}$, may in principle depend on the number of times of "going around" the orbit. The coefficients $a_{j, \gamma, k}$ are distributions on $\mathbb{R}: \mathcal{C}_{\mathrm{c}}^{\infty}(\mathbb{R}) \ni g \mapsto a_{j, \gamma, k}$.

It is now standard that under the non-degeneracy and simplicity assumptions, $\operatorname{det}\left(I-P_{\gamma}\right) \neq 0, T_{\gamma}=T_{\gamma^{\prime}} \Rightarrow \gamma=\gamma^{\prime}$, we can recover the actions, $S_{\gamma}$, periods, $T_{\gamma}$, Maslov indices, $\nu_{\gamma, k}$, and $\left|\operatorname{det}\left(I-P_{\gamma}^{k}\right)\right|$, for all $k$. Under stronger non-degeneracy conditions (see (1.4) and (2.9) below), a theorem of Fried [6] (see Proposition 3.2 below) then shows that the eigenvalues of the linear Poincaré map, $P_{\gamma}$, can be recovered.

The higher order coefficients $a_{j, \gamma, k}$ contain further information and they form a family of semi-classical spectral invariants. It turns out that under a stronger version of the non-degeneracy hypothesis, we can recover from them the full infinitesimal information about the non-linear Poincaré map $\kappa_{\gamma}$. For simplicity we state it here in the case of elliptic orbits only, which is the semi-classical analogue of Guillemin's result [8] - see Section 5 for the general statement. 
Thus suppose that the eigenvalues of $d \kappa_{\gamma}\left(w_{0}\right), w_{0}=U \cap \gamma$, are given by $\exp \left( \pm i \theta_{j}\right), j=1, \cdots, n, \theta_{j} \in(0, \pi)$. We strengthen the non-degeneracy assumption to independence from $2 \pi$ over rationals:

$$
\sum_{j=1}^{n} k_{j} \theta_{j} \in 2 \pi \mathbb{Z}, \quad k_{j} \in \mathbb{Z} \Longrightarrow k_{j}=0, j=1, \cdots, n .
$$

The now classical normal form theorem of Birkhoff, Lewis and Sternberg says that there exist symplectic coordinates $(x, \xi) \in \mathbb{R}^{2 n}$, centered at $w_{0}$, such that in a neighbourhood of $w_{0}$, we have

$$
\begin{gathered}
\kappa_{\gamma}=\kappa_{0}+\kappa^{b}, \quad \kappa^{b}(x, \xi)=\mathcal{O}\left(\left(x^{2}+\xi^{2}\right)^{\infty}\right) \\
\kappa_{0}(\iota, \omega)=\left(\iota, \omega-\frac{\partial p}{\partial \iota}\right), \quad \iota=\left(\iota_{1}, \cdots, \iota_{n}\right), \omega=\left(\omega_{1}, \cdots, \omega_{n}\right), \\
\iota_{j}=\frac{1}{2}\left(x_{j}^{2}+\xi_{j}^{2}\right), \quad \omega_{j}=\arg \left(x_{j}+i \xi_{j}\right), \\
p(\iota)=p(0)-\sum_{j=1}^{n} \theta_{j} \iota_{j}+\mathcal{O}\left(\iota^{2}\right),
\end{gathered}
$$

see [8, Theorem 2.1]. The Taylor series of the twist map $\kappa_{0}$ is called the Birkhoff normal form of $\kappa$. It invariantly describes the infinitesimal properties of the flow near $\gamma$. Since we chose $\theta_{j}$ 's in a unique way, $0<\theta_{j}<\pi$, the Birkhoff normal form is unique.

Specialized to this case, our general result, Theorem 4, can be stated as:

Theorem 1. Suppose that $P=-h^{2} \Delta+V(x)-E$ is a semi-classical Schrödinger operator satisfying (1.2), and that $\gamma$ is an elliptic closed orbit of $H_{p}$ on $p^{-1}(0)$, with eigenvalues of $P_{\gamma}, e^{ \pm i \theta_{j}}$, satisfying (1.4). Then the leading term and the coefficients $a_{j, \gamma, k}$ in (1.3), determine the Birkhoff normal form of $\kappa_{\gamma}$.

As stated above, our motivation for the recovery of the classical Birkhoff normal forms came from an attempt to understand the results of Guillemin [8] and Zelditch [20],[21],[22], in the context of the recent trace formula of SjöstrandZworski [18], and of the (quantum) Birkhoff normal form for semi-classical Fourier integral operator of Iantchenko [12] and Iantchenko-Sjöstrand [13] (see also [17]). Guillemin and Zelditch considered the high energy (or $\mathcal{C}^{\infty}$ singularities) case, corresponding to $h^{2} \Delta_{g}-1$ semi-classically. Guillemin's starting point was his earlier work with Françoise [5], and an observation of Zelditch on the non-commutative residues of (classical) Fourier integral operator [7]. Zelditch's approach also used the non-commutative residue but was more concrete and computational.

In addition to recovering the classical Birkhoff normal form, [8], [20], [21], [22] were concerned with recovering the full quantum Birkhoff normal form of the operator near the closed trajectory. Without giving a definition of the Birkhoff normal form this result follows from: 
Theorem 2. Suppose that the assumptions of Theorem 1 are satisfied for potentials $V$ and $\tilde{V}$, with orbits $\gamma$ and $\tilde{\gamma}$, respectively. If, in the notation of (1.3), $S_{\gamma}=\tilde{S}_{\tilde{\gamma}}, T_{\gamma}=\widetilde{T}_{\tilde{\gamma}}$, and $\left|\operatorname{det}\left(I-P_{\gamma}^{k}\right)\right|=\left|\operatorname{det}\left(I-\widetilde{P}_{\tilde{\gamma}}^{k}\right)\right|, \nu_{\gamma, k}=\tilde{\nu}_{\tilde{\gamma}, k}$, for all $k$, then

$\forall j, k, a_{j, \gamma, k}=\tilde{a}_{j, \tilde{\gamma}, k} \Longleftrightarrow\left\{\begin{array}{l}\text { There exists a unitary } h \text {-Fourier integral operator } \\ F, \text { microlocally defined in a neighbourhood of } \gamma, \\ \text { such that }\left(-h^{2} \Delta+\widetilde{V}\right) F=F\left(-h^{2} \Delta+V\right), \\ \text { microlocally to infinite order along } \gamma .\end{array}\right.$

The general result follows from Proposition 4.1 and Theorem 4 below, and is given in Corollary 5.2. The definitions of $h$-Fourier integral operators, and of agreement "to infinite order" are recalled in Section 2.

Our approach is a straightforward application of (2.3) and (2.13), quoted below from our earlier papers, and of a result of Fried [6] on the recovery of the eigenvalues of $P_{\gamma}$ (see Proposition 3.2 below). The trace formula shows that the traces of powers of the quantum monodromy operator of [18] are spectral invariants. The quantum monodromy operator is a semi-classical operator which quantizes the Poincaré map for a given orbit $\gamma$, and once it is put into the Birkhoff normal form using (2.13) the traces of its powers are easy to compute - see Proposition 3.1. The recovery of the classical Birkhoff normal form is then very clear - see Theorem 3.

One of the most striking applications of this approach to inverse problems is the result of Zelditch [22] on the recovery of bi-axial analytic planar domains from the spectrum of the (Dirichlet or Neumann) Laplacian. By applying our general methods, we avoid any specific new work involving the boundary value problem - see Theorem 5 and Corollary 5.4. This and other inverse results are presented in Section 5.

What may seem like an excessive generality in the trace formulæ of [18] (see (2.3) below) and Theorem 4 is motivated by their potential use for the recovery of classical dynamics of effective Hamiltonians. One of the most impressive inverse procedures is the application of the Onsager rules for determining Fermi surfaces - see for instance [1] for an introductory physics discussion. They can be interpreted in terms of a trace formula involving an effective Hamiltonian coming from the Peierls substitution - see [10]. The question which will be investigated elsewhere is whether or not more detailed information about the Fermi surface can be obtained from higher order terms in the expansions of magnetic susceptibilities of metals.

Throughout the paper, we will write

$$
u(x, \epsilon)=\sum_{j=0}^{\infty} u_{j}(x) \epsilon^{j},
$$

to denote asymptotic expansions as $\epsilon \rightarrow 0$. When no confusion is likely to arise all equalities are meant modulo $\mathcal{O}\left(\epsilon^{\infty}\right)$ where $\epsilon$ is the relevant small parameter (mostly $h$, the mathematical "Planck constant"). We denote by $\mathbb{N}$ the set of natural numbers, $0,1,2, \ldots$ 


\section{Preliminaries}

In this section we will recall the general trace formula of [18] and the classical and quantum Birkhoff normal forms of [13].

We consider $X$ which is either a compact $\mathcal{C}^{\infty}$ manifold of dimension $n+1$ or $\mathbb{R}^{n+1}$. Then we introduce the usual class of semi-classical symbols on $X$ :

$$
S^{m, k}\left(T^{*} X\right)=\left\{a \in \mathcal{C}^{\infty}\left(T^{*} X \times(0,1]\right):\left|\partial_{x}^{\alpha} \partial_{\xi}^{\beta} a(x, \xi ; h)\right| \leq C_{\alpha, \beta} h^{-m}\langle\xi\rangle^{k-|\beta|}\right\},
$$

and the corresponding class of pseudodifferential operators, $\Psi_{h}^{m, k}(X)$, with the quantization and symbol maps:

$$
\begin{aligned}
& \mathrm{Op}_{h}^{w}: S^{m, k}\left(T^{*} X\right) \longrightarrow \Psi_{h}^{m, k}(X) \\
& \sigma_{h}: \Psi_{h}^{m, k}(X) \longrightarrow S^{m, k}\left(T^{*} X\right) / S^{m-1, k-1}\left(T^{*} X\right),
\end{aligned}
$$

with both maps surjective, and the usual properties

$$
\begin{gathered}
\sigma_{h}(A \circ B)=\sigma_{h}(A) \sigma_{h}(B), \\
0 \rightarrow \Psi^{m-1, k-1}(X) \hookrightarrow \Psi^{m, k}(X) \stackrel{\sigma_{h}}{\rightarrow} S^{m, k}\left(T^{*} X\right) / S^{m-1, k-1}\left(T^{*} X\right) \rightarrow 0,
\end{gathered}
$$

a short exact sequence, and

$$
\sigma_{h} \circ \mathrm{Op}_{h}^{w}: S^{m, k}\left(T^{*} X\right) \longrightarrow S^{m, k}\left(T^{*} X\right) / S^{m-1, k-1}\left(T^{*} X\right),
$$

the natural projection map.

Let $P(z) \in \mathcal{C}^{\infty}\left(I_{z} ; \Psi_{h}^{0, k}(X)\right), I=(-a, a) \subset \mathbb{R}$, be a family of self-adjoint, principal type operators, such that $\Sigma_{z}=\{m: \sigma(P(z))=0\} \subset T^{*} X$ is compact. We assume that

$$
\sigma\left(\partial_{z} P(z)\right) \leq-C<0, \text { near } \Sigma_{z}, \quad|\sigma(P(z))| \geq C\langle\xi\rangle^{k},
$$

for $|\xi| \geq C$ when $X$ is a compact manifold, and for $|(x, \xi)| \geq C$ if $X=\mathbb{R}^{n+1}$.

In the example discussed in Section 1 , we have $X=\mathbb{R}^{n+1}$, and $P(z)=-h^{2} \Delta+$ $V(x)-E-z, z$ near 0 .

We also assume that for $z$ near 0 , the Hamilton vector field, $H_{p(z)}, p(z)=$ $\sigma(P(z))$, has a simple closed orbit $\gamma(z) \subset \Sigma_{z}$ with period $T(z)$, and that $\gamma(z)$ has a neighbourhood $\Omega$ such that

$$
\begin{aligned}
& m \in \Omega \text { and } \exp t H_{p(z)}(m)=m, p(z, m)=0, \\
& \qquad<|t| \leq T(z) N+\epsilon, z \in I, \Longrightarrow m \in \gamma(z),
\end{aligned}
$$

where $T(z)$ is the period of $\gamma(z)$, assumed to depend smoothly on $z$. We also write $p(z, m)$ for $\sigma(P(z))(m)$. Let $A \in \Psi_{h}^{0,0}(X)$ be a microlocal cut-off to a sufficiently small neighbourhood of $\gamma(0)$.

Then if $P(z)$ is an almost analytic extension of $P(z), z \in \mathbb{R}, \chi \in \mathcal{C}_{\mathrm{c}}^{\infty}(I)$, $\tilde{\chi} \in \mathcal{C}_{\mathrm{c}}^{\infty}(\mathbb{C})$, its almost analytic extension, $f \in \mathcal{C}^{\infty}(\mathbb{R})$, and 
supp $\hat{f} \subset\left(-N\left(C_{p}-\epsilon\right)+C, N\left(C_{p}-\epsilon\right)-C\right) \backslash\{0\}$, we have,

$$
\begin{gathered}
\frac{1}{\pi} \operatorname{tr} \int f(z / h) \bar{\partial}_{z}\left[\tilde{\chi}(z) \partial_{z} P(z) P(z)^{-1}\right] A \mathcal{L}(d z)= \\
-\frac{1}{2 \pi i} \sum_{-1 \neq k=-N-1}^{N-1} \operatorname{tr} \int_{\mathbb{R}} f(z / h) M(z, h)^{k} \frac{d}{d z} M(z, h) \chi(z) d z+\mathcal{O}\left(h^{\infty}\right),
\end{gathered}
$$

where $M(z, h)$ is the quantum monodromy operator. The constant $C_{p}>0$, in the condition on $\hat{f}$ depends on $p(z)$.

The quantum monodromy operator, $M(z, h)$ is defined as follows. For a point on $\gamma, m_{0} \in \gamma$, we can define the microlocal kernel of $P(z)$ at $m_{0}$, to be the set of families $u(h)$, such that $u(h)$ are microlocally defined near $m_{0}$ and $P(z) u(h)=$ $\mathcal{O}\left(h^{\infty}\right)$ near $m_{0}$. We denote it by $\operatorname{ker}_{m_{0}(z)}(P(z))$. Any solution can be continued microlocally along $\gamma(z)$ and we denote the corresponding forward and backward continuations by $I_{ \pm}(z)$ - see $[18, \S 4]$ for precise definitions. Now, let $m_{1} \neq m_{0}$ be another point on $\gamma(z)$. We then define

$$
\begin{gathered}
I_{-}(z) \mathcal{M}(z)=I_{+}(z), \quad \text { near } m_{1}, \\
\mathcal{M}(z): \operatorname{ker}_{m_{0}(z)}(P(z)) \longrightarrow \operatorname{ker}_{m_{0}(z)}(P(z)) .
\end{gathered}
$$

The operator $P(z)$ is assumed to be self-adjoint with respect to some inner product $\langle\bullet, \bullet\rangle$, and we define the quantum flux norm on $\operatorname{ker}_{m_{0}(z)}(P(z))$ as follows: let $\chi$ be a microlocal cut-off function supported near $\gamma$ and equal to one near the part of $\gamma$ between $m_{0}$ and $m_{1}$, in the positive direction determined by $H_{p}$. We denote by $[P(z), \chi]_{+}$the part of the commutator microlocally supported near $m_{0}$, and put

$$
\langle u, v\rangle_{\mathrm{QF}} \stackrel{\text { def }}{=}\left\langle[(i / h) P(z), \chi]_{+} u, v\right\rangle, \quad u, v \in \operatorname{ker}_{m_{0}(z)}(P(z)) .
$$

It is easy to check that this norm is independent of the choice of $\chi$ - see $[18$, Lemma 4.4]. This independence leads to the unitarity of $\mathcal{M}(z)$ :

$$
\langle\mathcal{M}(z) u, \mathcal{M}(z) u\rangle_{\mathrm{QF}}=\langle u, u\rangle_{\mathrm{QF}}, \quad u \in \operatorname{ker}_{m_{0}(z)}(P(z)) .
$$

Since microlocally, near a given point, the operator $P(z)$ can be reduced to $h D_{x_{1}}$ (see (4.1) below) we can identify $\operatorname{ker}_{m_{0}(z)}(P(z))$ with $\mathcal{D}^{\prime}\left(\mathbb{R}^{n}\right)$. This is done microlocally near $(0,0)$, and we can choose the identification so that the corresponding monodromy map is unitary (microlocally near $(0,0)$ where $(0,0)$ corresponds to the closed orbit intersecting a transversal identified with $T^{*} \mathbb{R}^{n}$ ). This gives

$$
M(z, h): \mathcal{D}^{\prime}\left(\mathbb{R}^{n}\right) \longrightarrow \mathcal{D}^{\prime}\left(\mathbb{R}^{n}\right)
$$

microlocally defined near $(0,0)$ and

$M(z, h)$ is a semi-classical Fourier integral operator which quantizes the Poincaré map of $\gamma(z)$. This, and (2.3), (2.6) are the only properties of $M(z, h)$ which will be used in this paper. 
The simplest example $P(z)=h D_{x}-z$, on $\mathbb{S}^{1}$ clarifies the basic notions reviewed above. In that case $M(z, h)=\exp (2 \pi i z / h)$ - see $[18, \S 2]$ for a careful presentation. In fact, the strategy of [18] is to apply microlocal techniques which essentially reduce the proof to that of the simplest case. For an interested reader we present, in the Appendix, a non-trivial example of $M(z, h)$. However, we stress that the abstract theory allows us to avoid any explicit computations until the final stage involving the analysis of the traces.

We will now assume that the symbol of $P(z)$ has an asymptotic expansion in powers of $h$, near $\Sigma_{0}$ :

$$
\begin{array}{r}
(P(z))(x, \xi ; h)=\sum_{j=0}^{\infty} h^{j}\left(p_{j}(z)\right)(x, \xi), \quad(x, \xi) \in U, \\
U \text { a precompact neighbourhood of } \Sigma_{0} .
\end{array}
$$

Under this assumption we have a simple extension of [18, Proposition 7.5]

$$
\operatorname{tr} M(z, h)^{k-1} h D_{z} M(z, h)=e^{i k I(z)} \frac{e^{i \nu_{k}(z) \frac{\pi}{2}} I^{\prime}(z)}{\left|d \kappa_{\gamma(z)}^{k}-1\right|^{\frac{1}{2}}}+e^{i k I(z)} \sum_{j=1}^{\infty} a_{j, k} h^{j},
$$

where $I(z)$ is the classical action, $\nu_{k}$ the Maslov index of the $k$ th iterate of $\gamma(z)$ (it is independent of $z$ for $z$ near 0 ).

We start by recalling the general statement of the classical Birkhoff normal form - see [13, Theorem 1.3, Proposition 4.3] and references there.

Let $(W, \omega)$ be a symplectic manifold of dimension $2 n$, and let $\kappa: W \longrightarrow$ $W, \quad \kappa\left(w_{0}\right)=w_{0}$, be a symplectic transformation, $\kappa^{*} \omega=\omega$, fixing $w_{0} \in W$. In (1.5), the twist map, $\kappa_{0}$, is the Birkhoff normal form of $\kappa$,

$$
\kappa_{0}=\exp H_{p}
$$

where the Hamiltonian $p$ is in the Birkhoff normal form.

The general case presented in [13] is formulated using (2.7): suppose that the eigenvalues of $d \kappa\left(w_{0}\right)$ are given by

$$
\begin{gathered}
\lambda_{j}, \lambda_{j}^{-1}, \quad j=1, \cdots, n \\
\lambda_{j}=\exp \mu_{j}, \quad \mu_{j}=\alpha_{j}+i \beta_{j}, \alpha_{j}>0,0<\beta_{j} \notin 2 \pi \mathbb{Z}, \quad 1 \leq j \leq n_{\mathrm{ch}}, \\
\lambda_{j+n_{\mathrm{ch}}}=\exp \mu_{j+n_{\mathrm{ch}}}, \mu_{j+n_{\mathrm{ch}}}=\overline{\mu_{j}}, \quad 1 \leq j \leq n_{\mathrm{ch}}, \\
\lambda_{j}=\exp \mu_{j}, \mu_{j}>0, \quad 2 n_{\mathrm{ch}}+1 \leq j \leq 2 n_{\mathrm{ch}}+n_{\mathrm{rh}}, \\
\lambda_{j}=\exp \mu_{j}, \quad \mu_{j} \in i(\mathbb{R} \backslash 2 \pi \mathbb{Z}), \quad n_{\mathrm{rh}}+2 n_{\mathrm{ch}}+1 \leq j \leq n .
\end{gathered}
$$

Here $n_{\mathrm{e}}+n_{\mathrm{rh}}+2 n_{\mathrm{ch}}=n$, and e stands for elliptic, rh for real hyperbolic, and ch, for complex hyperbolic.

We replace the condition in the purely elliptic case (1.4) by the more general condition

$$
\sum_{j=1}^{n} k_{j} \mu_{j} \in 2 \pi i \mathbb{Z}, k_{j} \in \mathbb{Z} \Longrightarrow k_{j}=0
$$


Then the generalization of (1.5) says that there exist symplectic coordinates $(x, \xi) \in \mathbb{R}^{n}$ centered at $(0,0)$

$$
\begin{aligned}
& \kappa=\exp H_{p}+\kappa^{b}, \quad \kappa^{b}(x, \xi)=\mathcal{O}\left(\left(x^{2}+\xi^{2}\right)^{\infty}\right), \\
& p=p_{0}+r, \quad r(x, \xi)=\mathcal{O}\left(\left(x^{2}+\xi^{2}\right)^{2}\right), \\
& p_{0}(x, \xi)=\sum_{j=1}^{n_{\mathrm{ch}}} \alpha_{j}\left(x_{2 j-1} \xi_{2 j-1}+x_{2 j} \xi_{2 j}\right)-\beta_{j}\left(x_{2 j-1} \xi_{2 j}-x_{2 j} \xi_{2 j-1}\right) \\
& +\sum_{j=2 n_{\mathrm{ch}}+1}^{2 n_{\mathrm{ch}}+n_{\mathrm{rh}}} \mu_{j} x_{j} \xi_{j}+\sum_{j=2 n_{\mathrm{ch}}+n_{\mathrm{rh}}+1}^{n} \frac{\mu_{j}}{2 i}\left(x_{j}^{2}+\xi_{j}^{2}\right) \\
& r(x, \xi)=R(\iota), \quad R(\iota)=\sum_{|\alpha| \geq 2} r_{\alpha} \iota^{\alpha}, \\
& \iota_{j}= \begin{cases}\left(x_{2 j-1} \xi_{2 j-1}+x_{2 j} \xi_{2 j}+i\left(x_{2 j-1} \xi_{2 j}-x_{2 j} \xi_{2 j-1}\right)\right) / 2 & 1 \leq j \leq n_{\mathrm{ch}} \\
\bar{\iota}_{j^{\prime}} & j^{\prime}=j-n_{\mathrm{ch}}, n_{\mathrm{ch}}+1 \leq j \leq 2 n_{\mathrm{ch}} \\
x_{j} \xi_{j} & 2 n_{\mathrm{ch}}+1 \leq j \leq 2 n_{\mathrm{ch}}+n_{\mathrm{rh}} \\
\left(x_{j}^{2}+\xi_{j}^{2}\right) /(2 i) & 2 n_{\mathrm{ch}}+n_{\mathrm{rh}}+1 \leq j \leq n\end{cases}
\end{aligned}
$$

Observe that $p_{0}=\langle\iota, \mu\rangle$. By the Birkhoff normal form of $\kappa$ we mean $p_{0}$ and the asymptotic expansion of $r$.

We now recall the quantum Birkhoff normal forms [12] and [13, Theorem 5.1, (4.11)]. We start with some general facts about $h$-Fourier integral operators. Let $W$ above be a neighbourhood of $w_{0} \in T^{*} X$, where $X$ is a $\mathcal{C}^{\infty}$ manifold. To $\kappa: W \rightarrow W$ we can associate a class of operators, microlocally defined near $w_{0}$ (see $[18, \S 3]$ for the precise definition of this notion):

$$
I_{h}^{m}\left(X, X, \kappa^{\prime}\right),
$$

the semi-classical Fourier integral operators quantizing $\kappa$. One way to define them is by considering oscillatory integrals: we identify $w_{0}$ with $(0,0) \in T^{*} \mathbb{R}^{n}$ so that

$$
\begin{gathered}
U \in I_{h}^{m}\left(X, X, \kappa^{\prime}\right) \Longleftrightarrow U u(x)=h^{-m-\frac{n+N}{2}} \iint e^{i \phi(x, y, \theta) / h} a(x, y, \theta ; h) u(y) d y d \theta, \\
\phi \in \mathcal{C}^{\infty}\left(\mathbb{R}^{n} \times \mathbb{R}^{n} \times \mathbb{R}^{N}\right), \quad \operatorname{graph}(\kappa)=\pi\left(C_{\phi}\right), \\
C_{\phi}=\left\{(x, y, \theta): \phi_{\theta}^{\prime}(x, y, \theta)=0\right\} \ni(x, y, \theta) \stackrel{\pi}{\longmapsto}\left(x, \phi_{x}^{\prime} ; y, \phi_{y}^{\prime}\right),
\end{gathered}
$$

where $\phi$ is assumed to be a non-degenerate phase function in the sense of Hörmander (see [11, Def. 21.2.5] for a related discussion), that is $\phi$ is smooth and real, $C_{\phi}$ is a smooth manifold of dimension $2 n$, and $\pi$ has an injective differential. The amplitude $a$ is assumed to be supported near $(0,0,0)$, and to be a classical symbol in the sense of (2.5).

Before stating the main result of [13] we have to introduce the notion of equivalence of families of operators used in that paper $[13, \S 2, \S 5]$ : let $U(z) \in$ 
$I^{0}\left(X, X, \kappa_{z}^{\prime}\right)$ and $\widetilde{U}(z) \in I^{0}\left(X, X, \tilde{\kappa}_{z}^{\prime}\right)$ be two families of operators. Then

$$
U \equiv \widetilde{U}
$$

if $\kappa_{0}\left(w_{0}\right)=\tilde{\kappa}_{0}\left(w_{0}\right)=w_{0}$, the two families of transformations, $\kappa_{z}$ and $\tilde{\kappa}_{z}$, agree to infinite order at $w_{0}$ and $z=0$, and the terms in the asymptotic expansions (in powers of $h$ ) of $\phi_{z}, \tilde{\phi}_{z}, a_{z}, \tilde{a}_{z}$ (with amplitudes as in (2.11)) agree to infinite order at $(0,0,0)$ and $z=0$.

Suppose that $U(z)$ quantizes $\kappa_{z}$ and $\kappa_{0}$ satisfies the assumptions of (2.10). Also assume that $U(z)$ is elliptic. Then

$$
\begin{gathered}
U(z) \equiv V(z)^{-1} e^{i P(z, h) / h} V(z), \\
V(z) \in I^{0}\left(X, X, \mathcal{C}_{z}^{\prime}\right)
\end{gathered}
$$

is a family of elliptic Fourier integral operators near $\left((0,0), w_{0}\right)$.

$$
\begin{gathered}
P(z, h)=p_{0}(z)+h p_{1}(z)+h^{2} p_{2}(z)+\cdots, \\
p_{j}(z)=p_{j}\left(z ; I_{1}, \cdots, I_{j}\right), \quad I_{j}=\iota_{j}^{w}\left(x, h D_{x}\right), \\
p_{0}(z)=\sum_{j=1}^{n} \mu_{j}(z) I_{j}+R\left(z ; I_{1}, \cdots, I_{n}\right), \quad R(z ; \iota)=\mathcal{O}\left(\iota^{2}\right) .
\end{gathered}
$$

When $U(z)$ is microlocally unitary near $w_{0}, P(z, h), V(z)$ can be chosen to be microlocally self-adjoint and unitary, respectively, at $\left((0,0), w_{0}\right)$. We will call the expansion of $P(z, h)$ at $z=0, \iota=0$, the infinitesimal Quantum Birkhoff normal form of $U(z)$ at $z=0$.

We recall that a Fourier integral operator is elliptic if it is associated to a canonical transformation, that is $\mathcal{C}$ above is the graph of a canonical transformation, $\left((0,0), w_{0}\right) \in \mathcal{C}$, and that its symbol is elliptic, that is the leading term in the asymptotic expansion of the amplitude in (2.11) does not vanish.

\section{Recovering Birkhoff normal forms from traces}

From [18, Lemma 3.2, Proposition 7.3] we know that if $U(z) \in I_{h}^{0}\left(X, X, \kappa(z)^{\prime}\right)$, is a family of $h$-Fourier integral operators, and that the canonical relations $\kappa(z)^{k}$, $k \neq 0$, have only one non-degenerate fixed point then

$$
\operatorname{tr} U(z)^{k}=e^{i k S(z) / h}\left(a_{0 k}(z)+a_{1 k}(z) h+\cdots+a_{l k}(z) h^{l}+\mathcal{O}\left(h^{l+1}\right)\right) .
$$

In this section we will prove the following:

Theorem 3. Let $U(z) \in I_{h}^{0}\left(X, X, \kappa(z)^{\prime}\right)$ be a family of $h$-Fourier integral operators associated to smooth locally defined canonical transformations, $\kappa(z)$ : $T^{*} X \rightarrow T^{*} X$. Suppose that $\kappa_{0}$ has a unique fixed point, $w_{0}$, and that the eigenvalues of $d \kappa_{0}\left(w_{0}\right)$ given in (2.8) satisfy (2.9). Then the Taylor series of the coefficients $a_{l k}(z)$ in (3.1) determine the infinitesimal quantum Birkhoff normal form of $U(z), P(z, h)$ in (2.13). In particular, the Birkhoff normal form of $\kappa_{0}$ at $z=0, p(z)$, in (2.10), is determined.

Our proof of Theorem 3 is based on: 
Proposition 3.1. Suppose that

$$
\begin{aligned}
G(\iota ; h) & =\langle\iota, \mu\rangle+F(\iota, h), \\
F(\iota, h) & =F_{0}(\iota)+h F_{1}(\iota)+\cdots, \quad e^{\mu_{j}} \neq 1, \\
F_{0}(\iota) & =\mathcal{O}\left(\iota^{2}\right),
\end{aligned}
$$

where we consider $F(\iota, h)$ as a formal power series in $h$, and each $F_{j}(\iota)$, a formal power series in $\iota$, and where we supressed the dependence on $\mu$ in higher order terms.

If $I_{j}(h)=\iota_{j}^{w}\left(x, h D_{x}\right)$, then

$$
\operatorname{tr} e^{-i G(I(h) ; h) / h}=e^{-i F\left(i h \partial_{\mu} ; h\right) / h}\left(\prod_{j=1}^{n} \frac{1}{2 \sinh \left(\mu_{j} / 2\right)}\right) .
$$

Proof. When $G(\iota ; h)=\langle\iota, \mu\rangle$ this follows from computations in each of the cases given in $(2.10)$ :

$$
\operatorname{tr} e^{-i\langle I(h), \mu\rangle / h}=\operatorname{tr} e^{-i\langle I(1), \mu\rangle},
$$

where we can separate the trace into separate blocks:

- elliptic blocks:

$$
\operatorname{tr} e^{-\mu\left(D_{x}^{2}+x^{2}\right) / 2}=\sum_{k=0}^{\infty} e^{-\mu\left(n+\frac{1}{2}\right)}=\frac{1}{2 \sinh \frac{\mu}{2}},
$$

where the formal computation $\left(\mu \in i \mathbb{R}_{+}\right)$can be justified by condsidering the limit $\epsilon \rightarrow 0$, with $\mu$ replaced by $\mu+\epsilon$.

- real hyperbolic blocks:

$$
\operatorname{tr} e^{-i \mu\left(x D_{x}+D_{x} x\right) / 2}=\operatorname{tr} e^{-\mu\left(x \partial_{x}+\frac{1}{2}\right)}=\operatorname{tr}\left(u \mapsto e^{-\mu / 2} u\left(e^{-\mu} \bullet\right)\right),
$$

Formally,

$$
\operatorname{tr} e^{-\mu\left(x \partial_{x}+\frac{1}{2}\right)}=e^{-\mu / 2} \int \delta_{0}\left(\left(e^{-\mu}-1\right) x\right) d x=\frac{1}{2 \sinh \frac{\mu}{2}},
$$

and this can be justified by approximating $\delta_{0}$ by a sequence of smooth compactly supported functions.

- complex hyperbolic blocks: here we have to work in two dimensions, $\mu_{1}=$ $\alpha+i \beta, \mu_{2}=\bar{\mu}_{1}=\alpha-i \beta$, and the corresponding $\iota_{1}$ and $\iota_{2}$ (see the last two lines in (2.10)) are $\iota_{1}=z \cdot \zeta, \iota_{2}=w \cdot \omega$, where $z=\left(x_{1}-i x_{2}\right) / \sqrt{2}$, $w=\bar{z}$ and $\zeta=\left(\xi_{1}+i \xi_{2}\right) / \sqrt{2}, \omega=\left(\xi_{1}-i \xi_{2}\right) / \sqrt{2}$, the corresponding dual coordinates, $\omega=\bar{\zeta}$. With $I_{j}=\iota_{j}^{w}\left(x, D_{x}\right), j=1,2$, we have

$$
\begin{aligned}
\operatorname{tr} e^{-i \mu_{1} I_{1}-i \mu_{2} I_{2}}=\operatorname{tr} e^{-\mu_{1}\left(z \partial_{z}+\frac{1}{2}\right)-\bar{\mu}_{1}\left(\bar{z} \partial_{\bar{z}}+\frac{1}{2}\right)} & \\
& =\operatorname{tr}\left(u(\bullet, \bar{\bullet}) \mapsto e^{-\mu_{1} / 2-\bar{\mu}_{1} / 2} u\left(e^{-\mu_{1}} \bullet, e^{-\bar{\mu}_{1}} \bar{\bullet}\right)\right)
\end{aligned}
$$


The last operator can be written as a contour integral,

$$
\begin{aligned}
& e^{-\mu_{1} / 2-\bar{\mu}_{1} / 2} u\left(e^{-\mu_{1}}, e^{-\bar{\mu}_{1}} \bar{z}\right) \\
& =\frac{1}{(2 \pi)^{2}} \iiint \int e^{i\left(\left(e^{-\mu_{1}} z-\tilde{z}\right) \tilde{\zeta}+\left(e^{-\bar{\mu}} \bar{z}-\tilde{w}\right) \tilde{\omega}\right)} u(\tilde{z}, \tilde{w}) d \tilde{z} d \tilde{w} d \tilde{\zeta} d \tilde{\omega}
\end{aligned}
$$

with the integration contour given by $\tilde{w}=\overline{\tilde{z}}, \tilde{\omega}=\overline{\tilde{\zeta}}$.

Formal stationary phase argument applied when taking the trace gives

$$
\prod_{j=1}^{2} \frac{1}{2 \sinh \frac{\mu_{j}}{2}} \text {. }
$$

Once we understand the contribution of the leading term, the following formal argument essentially gives (3.3). Since $I(h)$ is unitarily equivalent to $h I(1)$ we see that

$$
\operatorname{tr} e^{-i G(I(h) ; h) / h}=\operatorname{tr} e^{-i G(h I(1) ; h) / h} .
$$

On the other hand, $I(1)=\partial_{\mu}\langle I(1), \mu\rangle$, and hence

$$
e^{-i G(h I(1) ; h) / h}=e^{-i F(h I(1) ; h) / h} e^{-i\langle I(1), \mu\rangle}=e^{-i F\left(i h \partial_{\mu} ; h\right) / h} e^{-i\langle I(1), \mu\rangle} .
$$

By moving differentiation outside of the trace we obtain the expansion (3.3).

We also need a result of Fried [6] used in the same context in [8]:

Proposition 3.2. If the assumption (2.9) holds then the set

$$
a_{0}^{k} \prod_{j=1}^{n} \sinh \left(k \mu_{j} / 2\right), \quad k \in \mathbb{N},
$$

determines $\exp \mu_{j}$ 's and $a_{0}$ uniquely.

Proposition 3.2 and an argument based on Kronecker's Theorem give the following inverse result crucial in the proof of Theorem 3 (see $[8, \S 8]$ for the original approach, and $[21, \S 6]$ for an approach similar to the one presented here):

Lemma 3.3. Suppose that $\mu_{j}$ satisfy (2.9). Then for any polynomial, $p(\xi)=$ $\sum_{|\alpha| \leq M} a_{\alpha} \xi^{\alpha}$, the coefficients $a_{\alpha}$ can be recovered from the asymptotics of

$$
p\left(i k^{-1} \partial_{\mu}\right) \prod_{j=1}^{n} \frac{1}{2 \sinh \left(k \mu_{j} / 2\right)}, \quad k \longrightarrow \infty .
$$

Proof. Using the argument of the proof of Proposition 3.1 we can rewrite (3.4) with $e_{0}=(1, \ldots, 1)$ :

$$
\begin{aligned}
p\left(i k^{-1} \partial_{\mu}\right) \prod_{j=1}^{n} \frac{1}{2 \sinh \left(k \mu_{j} / 2\right)} & =p\left(i k^{-1} \partial_{\mu}\right) \sum_{m \in \mathbb{N}^{n}} e^{-\left\langle m+e_{0} / 2, k \mu\right\rangle} \\
& =\sum_{m \in \mathbb{N}^{n}} p\left(\left(m+e_{0} / 2\right) / i\right) e^{-\left\langle m+e_{0} / 2, k \mu\right\rangle} .
\end{aligned}
$$


To justify this expression we will consider it as a distribution obtained as a boundary value of a holomorphic function. We introduce new complex variables $z \in \Omega_{1}=D(0,1)^{n_{\mathrm{e}}}$ and $w \in \Omega_{2}=(D(0,1) \backslash 0)^{n_{\mathrm{ch}}}$ in a polydisc and a punctured polydisc respectively: at the boundary, $z_{j}=e^{i \mu_{j} / 2}$, and $w_{j}=e^{i \beta_{j+n_{\mathrm{e}}+n_{\mathrm{rh}}} / 2}$. We then write

$$
\begin{gathered}
m=\left(m_{\mathrm{ch}}^{\prime}, m_{\mathrm{ch}}^{\prime \prime}, m_{\mathrm{rh}}, m_{\mathrm{e}}\right) \in \mathbb{N}^{n}, \quad e_{0}=\left(e_{0}^{\mathrm{ch}}, e_{0}^{\mathrm{ch}}, e_{0}^{\mathrm{rh}}, e_{0}^{\mathrm{e}}\right), \\
\mu=\left(\mu_{\mathrm{ch}}, \bar{\mu}_{\mathrm{ch}}, \mu_{\mathrm{rh}}, \mu_{\mathrm{e}}\right), \quad \mu_{\mathrm{ch}}=\alpha+i \beta, \quad \mu_{\mathrm{rh}} \in \mathbb{R}_{+}^{n_{\mathrm{rh}}}, \quad \mu_{\mathrm{e}} \in(i \mathbb{R})^{n_{\mathrm{e}}}, \\
\left\langle m+e_{0} / 2, \mu\right\rangle=\left\langle n_{2}+e_{0}^{\mathrm{ch}}, \alpha\right\rangle+\left\langle n_{1}+e_{0}^{\mathrm{rh}} / 2, \mu_{\mathrm{rh}}\right\rangle+i\left\langle l_{1}+e^{\mathrm{e}} / 2, \mu_{\mathrm{e}} / i\right\rangle+i\left\langle l_{2}, \beta\right\rangle, \\
n_{2}=m_{\mathrm{ch}}^{\prime}+m_{\mathrm{ch}}^{\prime \prime}, \quad l_{2}=m_{\mathrm{ch}}^{\prime}-m_{\mathrm{ch}}^{\prime \prime}, \quad n_{1}=m_{\mathrm{rh}}, \quad l_{1}=m_{\mathrm{e}} .
\end{gathered}
$$

Here $m \in \mathbb{N}^{n}$ is arbitrary, and

$$
n_{1} \in \mathbb{N}^{n_{\mathrm{rh}}}, \quad l_{1} \in \mathbb{N}^{n_{\mathrm{e}}}, \quad n_{2} \in \mathbb{N}^{n_{\mathrm{ch}}}, \quad l_{2} \in \mathbb{Z}^{n_{\mathrm{ch}}},
$$

with the constraint that

$$
\frac{n_{2} \pm l_{2}}{2} \in \mathbb{N}^{n_{\mathrm{ch}}}
$$

which implies that $\left|l_{2}\right| \leq n_{2}$, component wise. We then put

$$
q(n, l)=p\left(\left(m+e_{0} / 2\right) / i\right), \quad z=e^{-\mu_{\mathrm{e}}} \in \mathbb{C}^{n_{\mathrm{e}}}, \quad w=e^{-i \beta / 2} \in \mathbb{C}^{n_{\mathrm{ch}}},
$$

(component-wise in the natural sense). In this notation (3.5) becomes

$$
\sum_{n \in \mathbb{N}^{n_{\mathrm{rh}}}+n_{\mathrm{ch}}} e^{-k\left(\left\langle n_{1}+e_{0}^{\mathrm{rh}} / 2, \mu_{\mathrm{rh}}\right\rangle+\left\langle n_{2}+e_{0}^{\mathrm{ch}} / 2, \alpha\right\rangle\right)} F_{n}\left(z^{k}, w^{k}\right),
$$

where

$$
F_{n}(z, w)=\sum_{\substack{l_{1} \in \mathbb{N}_{\mathrm{e}}, l_{2} \in \mathbb{Z}^{n_{\mathrm{ch}}} \\ \frac{n_{2} \pm l_{2}}{2} \in \mathbb{N}^{n} \mathrm{ch}}} q(n, l) z^{2 l_{1}+e_{0}^{\mathrm{e}}} w^{2 l_{2}},
$$

$(z, w) \in \Omega_{1} \times \Omega_{2} \subset \mathbb{C}^{n_{\mathrm{e}}} \times \mathbb{C}^{n_{\mathrm{ch}}}$. Here we note that for any fixed $n, q(n, l)$ is nonzero for finitely many $l_{2}$ 's and hence $F_{n}(z, w)$ is holomorphic in the punctured polydisc. Our task to recover the coefficients $q(n, l)$ from asymptotics as $k \rightarrow 0$. That amounts to recovering the holomorphic functions $F_{n}$. We observe that (3.5) shows that the distributional boundary values $F_{n}$ 's are smooth away from $z_{j}^{4}=1$.

An application of Kronecker's theorem ${ }^{1}$ shows that for any

$$
(x, y) \in(\partial D(0,1))^{n_{\mathrm{e}}} \times(\partial D(0,1))^{n_{\mathrm{ch}}},
$$

there exist a sequence $k_{r} \rightarrow \infty$ such that $\left(z^{k_{r}}, w^{k_{r}}\right) \rightarrow(x, y)$.

From (2.9) we deduce that

$$
\sum_{i=1}^{n_{\mathrm{rh}}+n_{\mathrm{ch}}} k_{j} \operatorname{Re} \mu_{j}=0 \Longrightarrow k_{j}=0 .
$$

\footnotetext{
${ }^{1}$ which says that if $\alpha \in \mathbb{Z}^{n}$ satisfies $\langle\alpha, k\rangle \in 2 \pi \mathbb{Z}, k \in \mathbb{Z}^{n}, \Rightarrow k=0$, then for any $y \in \mathbb{R}^{n}$ and $\epsilon>0$, there exists $N \in \mathbb{N}$ and $K \in \mathbb{Z}^{n}$, such that $|y-N \alpha-2 \pi K|<\epsilon$. Its use is justified by $(2.9)$.
} 
Hence by choosing sequences of $k \rightarrow \infty$, we can determine the coefficients, $F_{n}(x, y)$ in the first sum (3.6) (the terms have different rates of exponential decay). From that we determine the holomorphic functions $F_{n}$, and the coefficients, $q(n, l)$.

Proof of Theorem 3. Applying [13, Theorem 3.2] and [13, Theorem 4.4] we see that, up to $\mathcal{O}\left(h^{\infty}\right)$ errors, we can write

$$
\operatorname{tr} U(z)^{k}=\operatorname{tr} e^{-k i G(I(h), z ; h) / h},
$$

where $G$ is as in Proposition 3.1. Hence,

$$
\operatorname{tr} U(z)^{k}=\left.e^{-i k F\left(i h k^{-1} \partial_{\mu}, z ; h\right) / h} \operatorname{tr} e^{-i\langle I(1), k \mu\rangle}\right|_{\mu=\mu(z)} .
$$

Recovering the Birkhoff normal form of $\kappa$ means recovering the Taylor series of $\langle\iota, \mu(z)\rangle+F_{0}(\iota, z)$ from the trace. We will actually recover the full infinitesimal quantum Birkhoff normal form, $\langle\iota, \mu(z)\rangle+F(\iota, z ; h)$.

We write

$$
\begin{gathered}
\frac{F(h y, z ; h)}{h}=\sum_{j=0}^{\infty} h^{j} f_{j}(z, y), \quad f_{j}(z, y)=\sum_{l=0}^{j+1} \sum_{|\alpha|=j+1-l} \frac{1}{\alpha !} \partial^{\alpha} F_{l}(z, 0) y^{\alpha} \\
f_{j}(z, y)=\sum_{k=0}^{\infty} f_{j k}(y) z^{k} .
\end{gathered}
$$

The polynomials $f_{j k}(y)$ clearly determine the formal series $F(\iota, z ; h)$.

Since

$$
\begin{aligned}
& \exp \left(-i k F\left(h i k^{-1} \partial_{\mu}, z ; h\right) / h\right)=\exp \left(-i k \sum_{0}^{\infty} h^{j} f_{j}\left(z, i k^{-1} \partial_{\mu}\right)\right)= \\
& e^{-i k f_{0}(z)}\left(1-i \sum_{j=1}^{\infty} h^{j} k f_{j}\left(z, i k^{-1} \partial_{\mu}\right)-\frac{1}{2 !}\left(\sum_{j=1}^{\infty} h^{j} k f_{j}\left(z, i k^{-1} \partial_{\mu}\right)\right)^{2}+\cdots\right),
\end{aligned}
$$

we see from (3.3), that the coefficient of $h^{0}$ in the expansion of the trace is given by

$$
\begin{gathered}
e^{-i k f_{00}}\left(\left(1-i \sum_{m=1}^{\infty} z^{m} k f_{0 m}-\frac{1}{2 !}\left(\sum_{m=1}^{\infty} z^{m} k f_{0 m}\right)^{2}+\cdots\right) \times\right. \\
\left(1-i \sum_{j=1}^{\infty} z^{j} k\left\langle i k^{-1} \partial_{\mu}, \mu^{(j)}(0)\right\rangle-\frac{1}{2 !}\left(\sum_{j=1}^{\infty} z^{j} k\left\langle i k^{-1} \partial_{\mu}, \mu^{(j)}(0)\right\rangle\right)^{2}+\cdots\right)^{\left.\operatorname{tr} e^{-i\langle I(1), k \mu\rangle}\right|_{\mu=\mu(0)}} \times
\end{gathered}
$$

where we expanded $\mu(z)$ into its Taylor series and then used the same argument as in the proof of Proposition 3.1. We apply Proposition 3.2 to the constant term 
to recover $\exp i f_{00}$ and $\mu(0)$. For $j>0$, the coefficient of $z^{j}$ in the expression above is given by

$$
-\left.i k e^{-i k f_{00}}\left(f_{0 j}+\left\langle k^{-1} i \partial_{\mu}, \mu^{(j)}(0)\right\rangle+H_{j, k}\left(i k^{-1} \partial_{\mu}\right)\right)\left(\prod_{j=1}^{n} \frac{1}{2 \sinh \left(k \mu_{j} / 2\right)}\right)\right|_{\mu=\mu(0)},
$$

where $H_{j, k}$ is constructed from $f_{0 l}$ 's and $\mu^{(p)}(0)$ with $l, p<m$. Hence Lemma 3.3 shows that $f_{0 j}$ 's and $\mu^{(j)}(0)$ can be successively recovered.

We now move to higher powers of $h$, with the procedure being essentially the same: we see from (3.7) that the coefficient of $h^{m}$ in the expansion of the trace is given by

$$
\left.e^{-i k f_{0}(z)}\left(-i k f_{m}\left(i k^{-1} \partial_{\mu}, z\right)+G_{m, k}\left(i k^{-1} \partial_{\mu}, z\right)\right)\left(\prod_{j=1}^{n} \frac{1}{2 \sinh \left(k \mu_{j} / 2\right)}\right)\right|_{\mu=\mu(z)},
$$

where $G_{m, k}$ is constructed from $f_{l}$ 's with $l<m$, and we already know the $z$ expansions of $f_{0}(z)$ and $\mu(z)$. Replacing $f_{m}$ by its expansion in $z$, reduces the problem to recovering the polynomials $f_{m l}(y)$ from

$$
f_{m l}\left(i k^{-1} \partial_{\mu}\right) \prod_{j=1}^{n} \frac{1}{2 \sinh \left(k \mu_{j} / 2\right)}, \quad k \in \mathbb{N},
$$

and that follows again from Lemma 3.3. This shows that we recovered the full expansion (in $\iota, z$, and $h$ ) of $G(\iota, z ; h)$, that is the infinitesimal quantum Birkhoff normal form at $z=0$.

\section{The monodromy operator determines $P$ near $\gamma$.}

The trace formula (2.6) and the results of Section 3 will be used to recover the infinitesimal quantum Birkhoff normal form of the quantum monodromy operator $M(z)$ at $z=0$. In this section we will discuss the recovery of the full Hamiltonian $P(z)$, infinitesimally at $z=0$, from $M(z)$. Since our information comes from the left hand side of the trace formula (2.6), this recovery can only be possible up to conjugation by families of elliptic $h$-Fourier integral operators, and up to multiplication by families of elliptic $h$-pseudo-differential operators. The assumptions (2.1), the implicit function theorem, and the usual symbolic iteration, imply that

$$
P(z)=A(z)^{*}(P-z) A(z),
$$

with $A(z) \in \Psi_{h}^{0, k / 2}(X)$ elliptic near $\gamma(0)$, and $P \in \Psi_{h}^{0,0}(X)$ is self-adjoint. Replacing $P(z)$ by $P-z$ in $(2.6)$ changes the trace by $\mathcal{O}\left(h^{\infty}\right)$. Hence we would like to recover $P$ infinitesimally near $\gamma$, on the energy surface $p^{-1}(0), p=\sigma(P)$.

Thus in this section, let $P$ be a self-adjoint $h$-pseudodifferential operator with leading symbol $p$. Let $\gamma$ be a simple closed $H_{p}$-trajectory of period $T_{0}>0$. Recall from $[18, \S 4]$ that the quantum time, $Q$, is an $h$-pseudodifferential operator 
microlocally defined in a suitable neighbourhood $W_{+}=\operatorname{neigh}\left(m_{0}\right)$ of some fixed point $m_{0} \in \gamma$, such that

$$
\frac{i}{h}[P, Q]=I d .
$$

After conjugation by a unitary Fourier integral operator, we may assume that

$$
P=h D_{x_{n}}, Q=x_{n} .
$$

Let $K(z): \mathcal{D}^{\prime}\left(\mathbb{R}^{n}\right) \rightarrow \mathcal{D}^{\prime}(X)$ be a microlocally defined Fourier integral operator with

$$
(P-z) K(z)=0, \quad \frac{i}{h} K(z)^{*}[P, \chi]_{+} K(z)=I d,
$$

where $\chi \in C^{\infty}\left(W_{+}\right)$is equal to 0 near the incoming part of $\gamma$ and equal to 1 near the outgoing part, and we let $\chi$ also denote a corresponding $h$-quantization. In the case of (4.1), we can take

$$
K(z) u(x)=e^{i z x_{n} / h} u\left(x^{\prime}\right), \quad x=\left(x^{\prime}, x_{n}\right) .
$$

It follows from the second part of (4.2), that

$$
K(z)^{*} f^{\prime}(Q) K(z)=I d,
$$

for $f \in C^{\infty}(\mathbb{R}),=0$ for large negative $t$ and $=1$ for large positive $t$. Indeed, $e^{i t P / h} Q e^{-i t P / h}=Q+t$ and

$$
\begin{aligned}
\frac{i}{h}[P, f(Q)] & =\left(\partial_{t}\right)_{t=0}\left(e^{i t P / h} f(Q) e^{-i t P / h}\right)=\left(\partial_{t}\right)_{t=0} f\left(e^{i t P / h} Q e^{-i t P / h}\right) \\
& =\left(\partial_{t}\right)_{t=0} f(Q+t)=f^{\prime}(Q) .
\end{aligned}
$$

Notice that $f^{\prime} \in C_{0}^{\infty}(\mathbb{R}), \int f^{\prime}(q) d q=1$. More generally,

$$
K(z)^{*} g(Q) K(z)=\int g(q) d q \cdot I d
$$

for $g \in C_{0}^{\infty}(\mathbb{R})$. As in [18, Proposition 4.3] we can choose $K(z)$ so that

$$
K(z)=e^{i(z-w) Q / h} K(w),
$$

since this is fulfilled when (4.1), (4.3) hold (note that for $P(z)=P-z, U(z, w)=$ $\exp (i(z-w) Q)$, in $[18,(4.4)])$.

Consider

$$
\pi(z) \stackrel{\text { def }}{=} \frac{1}{2 \pi h} K(z) K(z)^{*} .
$$

Notice that this definition is independent of the choice of $K(z)$ satisfying (4.2).

We have the properties:

$$
\begin{gathered}
\pi(z)=\pi(z)^{*}, \\
P \pi(z)=\pi(z) P=z \pi(z), \\
\int \pi(z) d z=I d,
\end{gathered}
$$


where for the understanding of (4.8), we remark that if $\phi \in C_{0}^{\infty}(\mathbb{R})$ is independent of $h$, then $\int \phi(z) \pi(z) d z$ is a well defined $h$-pseudodifferential operator of order 0 , and that we can always choose $\phi$ to be equal to 1 in some interval corresponding to the region of phase space where we wish to establish (4.8).

The relations (4.6), (4.7) are obvious, and so is (4.8) if we work in the representation (4.1), (4.3).

We also give a direct proof of (4.8) which does not involve conjugation to the model: Let $g \in C_{0}^{\infty}(\mathbb{R})$ and consider

$$
K(z)^{*} g(Q) K(w)
$$

where we will assume (4.5). Then we get

$$
K(z)^{*} g(Q) K(w)=K^{*}(z) g(Q) e^{-i(z-w) Q / h} K(z)=\left(\mathcal{F}_{h} g\right)(z-w) \cdot I d,
$$

where $\mathcal{F}_{h} g(z)=\int e^{-i q z / h} g(q) d q$ is the $h$-Fourier transform. Here the second equality follows from (4.4).

Consider the microlocally defined $h$-pseudodifferential operator:

$$
A=\int \pi(z) d z
$$

$A$ is an elliptic pseudodifferential operator of order 0 , and we claim that

$$
A^{2}=A \text {. }
$$

Since the statement is microlocal, it suffices to show that

$$
A g(Q) A=A g(Q)
$$

$g \in C_{0}^{\infty}$ (and then choose $g(Q)=1$ in the region of interest). To get (4.11), we first compute:

$$
\begin{aligned}
\pi(z) g(Q) \pi(w) & =\frac{1}{(2 \pi h)^{2}} K(z) K(z)^{*} g(Q) K(w) K(w)^{*} \\
& =\frac{1}{(2 \pi h)^{2}} K(z) K(z)^{*}\left(\mathcal{F}_{h} g\right)(z-w) e^{i(z-w) Q / h},
\end{aligned}
$$

where we used (4.9), (4.4). Integrating with respect to $w$, we get

$$
\pi(z) g(Q) A=\pi(z) g(Q)
$$

which gives (4.10) after integration in $z$.

Rewriting (4.10) as $A(A-1)=0$ and using that $A$ is elliptic, we get (4.8).

Remark. Using (4.3) (or a direct argument), one can check that

$$
\pi(z)=\frac{1}{2 \pi h} \int e^{i t(P-z) / h} d t
$$

where the integral to the right is microlocally well-defined by restricting the integration to a suitable finite interval.

Let $P, \widetilde{P}$ be two $h$-pseudodifferential operators with simple closed trajectories

$$
\gamma \subset p^{-1}(0), \quad \widetilde{\gamma} \subset \widetilde{p}^{-1}(0)
$$


with the same period $T_{0}$. Let $m_{0} \in \gamma, \widetilde{m}_{0} \in \widetilde{\gamma}$ and assume that the corresponding monodromy operators

$$
\begin{aligned}
& \mathcal{M}(z): \operatorname{ker}_{m_{0}}(P-z) \longrightarrow \operatorname{ker}_{m_{0}}(P-z), \\
& \widetilde{\mathcal{M}}(z): \operatorname{ker}_{\tilde{m}_{0}}(\widetilde{P}-z) \longrightarrow \operatorname{ker}_{\tilde{m}_{0}}(\widetilde{P}-z),
\end{aligned}
$$

are conjugated:

$$
\mathcal{U}(z)^{-1} \widetilde{\mathcal{M}}(z) \mathcal{U}(z)=\mathcal{M}(z)
$$

where $\mathcal{U}(z): \operatorname{ker}_{\tilde{m}_{0}}(\widetilde{P}-z) \longrightarrow \operatorname{ker}_{m_{0}}(P-z)$ is a unitary Fourier integral operator depending smoothly on $z$.

Identifying $P, \widetilde{P}$ with $h D_{x_{n}}$, we see that

$$
F \stackrel{\text { def }}{=} \int \mathcal{U}(z) \pi(z) d z
$$

is an elliptic unitary Fourier integral operator with

$$
F_{\left.\right|_{\operatorname{ker}_{m_{0}}(P-z)}}=\mathcal{U}(z), \quad \widetilde{P} F=F P .
$$

We can identify $P, \widetilde{P}$ with $h D_{x_{n}}$ in such a way that $F$ becomes the identity operator. With this identification, we take $K(z)=\widetilde{K}(z)$ of the form (4.3). Without using the identification, this means that $\widetilde{K}(z)=F K(z)$ and

$$
F=\frac{1}{2 \pi h} \int \widetilde{K}(z) K(z)^{*} d z .
$$

Also,

$$
\widetilde{M}(z)=M(z)
$$

Let $K_{f}(z), \widetilde{K}_{f}(z) ; K_{b}(z), \widetilde{K}_{b}(z)$ be the forward and backward extensions of $K ; \widetilde{K}$ as in $[18, \S 5]$.

If $u$ is microlocally concentrated near $m=\exp \left(t H_{p}\right)\left(m_{0}\right),-\epsilon \leq t \leq T_{0}-2 \epsilon$, where $T_{0}$ is the common period of $\gamma, \tilde{\gamma}$, we define $F_{f} u$, microlocally concentrated to a neighbourhood of $\exp \left(t H_{p}\left(\widetilde{m}_{0}\right)\right)$, by

$$
F_{f} u=e^{-i t \tilde{P} / h} F e^{i t P / h} u .
$$

Since $F$ intertwines $\widetilde{P}$ and $P$, this definition is invariant under small variations of $t$ and we see that $F_{f}$ is a unitary Fourier integral operator with canonical transformation, given by

$$
\kappa_{F_{f}}(m)=\exp \left(t H_{\tilde{p}}\right) \circ \kappa_{F} \circ \exp \left(-t H_{p}\right)(m),
$$

for $m$ in a neighbourhood of $\exp \left(t H_{p}\right)\left(m_{0}\right)$, and where $\kappa_{F}:$ neigh $\left(m_{0}\right) \rightarrow$ neigh $\left(\widetilde{m}_{0}\right)$ is the canonical transformation associated to $F$.

We have

$$
F_{f} u=\frac{1}{2 \pi h} \int \widetilde{K}_{f}(z) K_{f}(z)^{*} d z
$$


In fact, by (4.13), (4.15):

$$
\begin{aligned}
F_{f} & =\frac{1}{2 \pi h} \int e^{-i t \tilde{P} / h} \widetilde{K}_{f}(z) K_{f}(z)^{*} e^{i t P / h} d z \\
& =\frac{1}{2 \pi h} \int e^{-i t z / h} \widetilde{K}_{f}(z) K_{f}(z)^{*} e^{i t z / h} d z=\frac{1}{2 \pi h} \int \widetilde{K}_{f}(z) K_{f}(z)^{*} d z .
\end{aligned}
$$

Similarly, if $u$ is microlocally concentrated near $m=\exp \left(t H_{p}\right)\left(m_{0}\right),-T_{0}+$ $2 \epsilon \leq t \leq \epsilon$, we define $F_{b} u$ to be the right hand side of (4.15) (now with $t$ in a different interval) and get

$$
F_{b} u=\frac{1}{2 \pi h} \int \widetilde{K}_{b}(z) K_{b}(z)^{*} d z
$$

If $m$ belongs to the overlap region, where both the forward and the backward extensions of $F$ are defined, then for $u$ concentrated to a neighbourhood of $m$, we have $F_{f} u=F_{b} u$ :

$$
F_{f} u=\frac{1}{2 \pi h} \int \widetilde{K}_{f}(z) K_{f}(z)^{*} u d z=\frac{1}{2 \pi h} \int \widetilde{K}_{b}(z) \widetilde{M}(z) M(z)^{*} K_{b}(z)^{*} u d z=F_{b} u,
$$

since $\widetilde{M}(z) M(z)^{*}=\widetilde{M}(z) M(z)^{-1}=1$, in view of (4.14).

This means that $F$ extends to a well-defined operator in a neighbourhood of $\gamma$, and we get:

Proposition 4.1. Let $P, \widetilde{P}$ be two h-pseudodifferential operators with closed simple trajectories $\gamma \subset p^{-1}(0), \widetilde{\gamma} \subset \widetilde{p}^{-1}(0)$ having the same period. Let $m_{0} \in \gamma$, $\widetilde{m}_{0} \in \widetilde{\gamma}$, and assume that the monodromy operators

$$
\begin{gathered}
\mathcal{M}(z): \operatorname{ker}_{m_{0}}(P-z) \longrightarrow \operatorname{ker}_{m_{0}}(P-z) \\
\widetilde{\mathcal{M}}(z): \operatorname{ker}_{\tilde{m}_{0}}(\widetilde{P}-z) \longrightarrow \operatorname{ker}_{\tilde{m}_{0}}(\widetilde{P}-z)
\end{gathered}
$$

are conjugate as in (4.12), via an $h$-Fourier integral operator $\mathcal{U}(z)$ depending smoothly on $z$. Then there exists a unitary Fourier integral operator F, microlocally defined in a neighbourhood of $\gamma$ with an associated canonical transformation $\kappa_{F}: \operatorname{neigh}(\gamma) \rightarrow \operatorname{neigh}(\widetilde{\gamma})$, such that $\widetilde{P} F=F P$.

Using Theorem 3, we will recover the infinitesimal quantum Birkhoff normal form of $\mathcal{M}(z)$ at $z=0$. From the proof of Proposition 4.1 it is clear that the conclusions remain valid to infinite order at $\gamma$ and $z=0$, if that assumption is made on the conjugation. Hence we obtain:

Proposition 4.2. Suppose that in Proposition 4.1 we only assume that

$$
\widetilde{\mathcal{M}}(z) \equiv \mathcal{U}(z) \mathcal{M}(z) \mathcal{U}(z)^{-1}
$$

to infinite order at $m=m_{0}, z=0$ in the sense of (2.12). Then there exists a unitary Fourier integral operator F, microlocally defined in a neighbourhood of $\gamma$ with an associated canonical transformation $\kappa_{F}:$ neigh $(\gamma) \rightarrow$ neigh $(\widetilde{\gamma})$, such that

$$
\widetilde{P} F=F P, \quad \text { to infinite order along } \gamma
$$




\section{Applications to inverse problems}

We start with a simple lemma motivated by (2.3) and (2.6):

Lemma 5.1. Let $u(z, h)$ be a semiclassical family of functions defined near $z=0$,

$$
u(z, h)=e^{i I(z) / h} \sum_{j=0}^{\infty} a_{j}(z) h^{j}, \quad I, a_{j} \in \mathcal{C}^{\infty}, a_{0}(0) \neq 0, \operatorname{Im} I(z)=0 .
$$

Suppose that $\mathcal{V} \subset \mathcal{C}_{\mathrm{c}}^{\infty}(\mathbb{R})$ is a subspace from which we can pick an element having any prescribed finite Taylor polynomial at $I^{\prime}(0)$. If for any $g \in \mathcal{V}$ we know $b_{j}(g)$ where

$$
\begin{aligned}
& J(g, u)=h^{-1} \int \hat{g}(z / h) \chi(z) u(z, h) d z= \\
& e^{i I(0) / h} \sum_{k=0}^{\infty} b_{k}(g) h^{k}, \quad \chi \in \mathcal{C}_{\mathrm{c}}^{\infty}(\mathbb{R}), \quad \chi \equiv 1 \text { near } 0,
\end{aligned}
$$

then the distributions $b_{k}$ determine the $I^{(l)}(0)$, and $a_{j}^{(l)}(0)$, for all $l$ and $j$.

Proof. We first observe that if $a_{j}(z)=\mathcal{O}\left(z^{\infty}\right)$ then they contribute $\mathcal{O}\left(h^{\infty}\right)$. Hence in considering the expansion of $J(g, u)$ we can replace $I$ and $a_{j}$ 's by their formal power expansions:

$$
I(z)=I_{0}+I_{1} z+\sum_{k=1}^{\infty} I_{k+1} z^{k+1}, \quad a_{j}(z)=\sum_{k=0}^{\infty} a_{j k} z^{k},
$$

and our task is the recovery of $I_{k}$ 's and $a_{j k}$ 's. Making a change of variables $\zeta=z / h$ we write $J(g, u)$ as the formal integral

$$
e^{I_{0} / h} \int_{\mathbb{R}} \hat{g}(\zeta) e^{i I_{1} \zeta} e^{i \sum{ }_{j=1}^{\infty} h^{j} I_{j+1} \zeta^{j+1}}\left(\sum_{j, k \geq 0} a_{j k} \zeta^{k} h^{k+j}\right) d \zeta,
$$

where we can neglect $\chi(h \zeta)$, as $\chi(z) \equiv 1$ near $z=0$. Just as in the proof of Theorem 3 we now see that the coefficient of $h^{p+1}$ in the expansion is given by

$$
e^{I_{0} / h} h \int \hat{g}(\zeta) e^{i I_{1} \zeta}\left(\sum_{l+k=p} a_{l k} \zeta^{l}+i a_{00} I_{p+1} \zeta^{p+1}+H_{m}(a, I)\right),
$$

where $H_{m}(a, I)$ depends only on $a_{l k}$ with $l+k<p$, and $I_{l}$ with $l<p+1$. Since we assumed that $a_{00}=a_{0}(0) \neq 0$, and that we can find $g \in \mathcal{V}$ with any prescribed finite Taylor polynomial at $I_{1}$, the coeffients of $I$ and $a$ can be recovered from $b_{j}(g)$ 's, $g \in \mathcal{V}$.

Using Lemma 5.1, and [18, Theorem 2, Proposition 7.5] (see (2.3) (2.6) above) we obtain the following consequence of Theorem 3 : 
Theorem 4. Suppose that $P(z)$ satisfies the assumptions needed for (2.3) and is classical in the sense of (2.5). Suppose that in addition the eigenvalues of the linear Poincaré map of $\gamma(0)$ satisfy (2.9). Then the coefficients in the asymptotic expansion of

$$
\frac{1}{\pi} \operatorname{tr} \int f(z / h) \bar{\partial}_{z}\left[\tilde{\chi}(z) \partial_{z} P(z) P(z)^{-1}\right] A \mathcal{L}(d z)=\sum_{k \in \mathbb{Z} \backslash\{0\}} e^{i k I / h} \sum_{j=0}^{\infty} a_{j, k}(f) h^{j},
$$

determine the infinitesimal quantum Birkhoff normal form of the quantum monodromy operator $M(z)$ at $z=0$. In particular the Birkhoff normal form of the Poincaré map of $\gamma(0)$ is determined. Here the notation is the same as in (2.3), and by the coefficients we mean the distributions, $f \mapsto a_{j}(f), \hat{f} \in \mathcal{C}_{\mathrm{c}}^{\infty}(\mathbb{R} \backslash\{0\})$.

Proof. We first see that the non-degeneracy of $\gamma(z)$ for $z$ close to 0 shows that (2.6) holds. We apply (2.3) and integrate by parts:

$$
\begin{aligned}
-\frac{1}{2 \pi i} \sum_{-1 \neq k=-N-1}^{N-1} \operatorname{tr} \int_{\mathbb{R}} f(z / h) M(z, h)^{k} \frac{d}{d z} M(z, h) \chi(z) d z= \\
h^{-1} \frac{1}{2 \pi i} \sum_{-1 \neq k=-N-1}^{N-1} \operatorname{tr} \int_{\mathbb{R}} f^{\prime}(z / h)(k+1)^{-1} M(z, h)^{k+1} \chi(z)+\mathcal{O}\left(h^{\infty}\right),
\end{aligned}
$$

where $\chi \equiv 1$ near 0 and hence the contribution of $\chi^{\prime}$ is negligible: $f(z / h)=$ $\mathcal{O}\left(h^{\infty}\right)$ for $|z|>\epsilon>0$. The assumptions of Lemma 5.1 with $\hat{g}=f^{\prime}$ are satisfied and hence we obtain expansions of $\operatorname{tr} M(z, h)^{k}$, for any $k$. Now we can apply Theorem 3 with $U(z)=M(z, h)$ and the proof is completed.

When we combine this result with Proposition 4.2 we obtain infinitesimal information about $P(z)$ at $\gamma$ :

Corollary 5.2. Suppose that the assumptions of Theorem 4 are satisfied and $P(z)=A(z)^{*}(P-z) A(z)$, where $A(z)$ is elliptic near $\gamma$. Then, up to conjugation by elliptic $h$-Fourier integral operators, in the sense of Proposition 4.2, $P$ is determined to infinite order at $\gamma$.

When specialized to the classical, or high energy, case, we obtain:

Corollary 5.3. Let $M$ be a compact $\mathcal{C}^{\infty}$ manifold and let $P$ be a positive, selfadjoint elliptic classical pseudo-differential operator acting on $\mathcal{C}^{\infty}\left(M, \Omega_{M}^{\frac{1}{2}}\right)$. Suppose that $\gamma \subset p^{-1}(1)$ is a simple non-degenerate orbit of the classical flow of the principal symbol of $P$, and $P_{\gamma}$ is its linear Poincaré map. If (2.9) holds for the eigenvalues of $P_{\gamma}(2.8)$, and no other orbit in $p^{-1}(1)$ has the period which is a multiple of the period of $\gamma$, then the spectrum of $P$ determines the Birkhoff normal form of the Poincaré first return map.

Proof. Suppose that the order of $P$ is $m$. We define a semi-classical operator, $P(h)$, by putting, $P(h)=h^{m} P\left(x, D_{x}\right)-1$, so that the (semi-classical) principal symbol of $P(h)+1$ is the same as the (classical) principal symbol of $P$. The non-degeneracy assumption for $\gamma \subset\{p=1\}$ implies that the assumptions of 
Theorem 4 are satisfied, with $P(z)=P(h)-z$. The simplicity of $\gamma$ then shows that for $f$ 's with supp $\hat{f}$ compact, and sufficiently close to $\mathbb{Z} T_{\gamma}$, where $T_{\gamma}$ is the (primitive) period of $\gamma$ on $\{p=1\}$, we have

$$
\operatorname{tr} f(P(h) / h) \chi(P(h)) A=\operatorname{tr} f(P(h) / h) \chi(P(h))+\mathcal{O}\left(h^{\infty}\right) .
$$

The left hand side is a (semi-classical) spectral invariant and hence the result follows from Theorem 4 .

We leave it to the reader to formulate the corresponding corollary in the more general semi-classical case.

In the case of manifolds with boundaries we consider, for the sake of simplicity, the case of second order differential operators only:

Theorem 5. Suppose that $(M, g)$ is a Riemannian manifold with boundary, and that $\Delta_{g}$ is its Dirichlet or Neumann Laplacian.

Let $\gamma$ be a simple non-degenerate orbit of the classical billiard flow of $g$. If all the intersections of $\gamma$ with $\partial M$ are transversal, (2.9) holds for eigenvalues of the Poincaré map of $\gamma(2.8)$, and the lengths of other closed orbits of the generalized billiard flow do not accumulate at a multiple of the period of $\gamma$, then the spectrum of $\Delta_{g}$ determines the Birkhoff normal form of the Poincaré first return map of $\gamma$.

Outline of the proof. First we need to indicate why the results of [18] can be applied in this situation. For that we will check that local solutions can be microlocally defined near a trajectory $\gamma$. Let $p$ be the symbol of $D_{t}^{2}-\Delta_{g}$ and $q$ the defining function of $\partial M$. The transversality condition on $\gamma$ means that $H_{q} p \neq 0$ at the boundary. We first recall (see for instance $[11, \S 24.2]$ ) that we can find coordinates in which, near a point in $\partial M, q=x_{1}$, and $p(x, \xi)=\xi_{1}^{2}-r\left(x, \xi^{\prime}\right)$, $\xi^{\prime}=\left(\xi_{2}, \cdots, \xi_{m+1}\right), m=\operatorname{dim} M$. Solving the boundary problem microlocally is equivalent to a hyperbolic problem and hence we have a solution. For localization needed in (2.3) we can use, near the boundary, an operator $A=A\left(x, h D_{x^{\prime}}\right){ }^{2}$

A more subtle argument is needed for showing that (5.1) holds, as that involves results on propagation of singularities for boundary value problems - see $[11, \S 24.5]$ and references given there. These results provide the foundation for the trace formula of Guillemin and Melrose [9] (see also [16]), which among other things shows that $\operatorname{singsupp}\left(\operatorname{tr} \cos \left(t \sqrt{-\Delta_{g}}\right)\right) \subset \overline{\mathcal{L}}$, where $\mathcal{L}$ is the set of length of closed orbits of the billiard flow. It also follows from [9] and [16] that if $A\left(x,\left\langle D_{t}\right\rangle^{-1} D_{x}\right)$ is an operator which provides a localization to a neighbourhood of the bicharacteristic strip of $\gamma$, then

$$
\operatorname{tr} \cos \left(t \sqrt{-\Delta_{g}}\right)-\operatorname{tr} \cos \left(t \sqrt{-\Delta_{g}}\right) A\left(x,\left\langle D_{t}\right\rangle^{-1} D_{x}\right) \in \mathcal{C}^{\infty}(\mathbb{R}), \quad \text { for } t \text { near } k T_{\gamma},
$$

\footnotetext{
${ }^{2}$ Alternatively, we could have reduced the problem to the boundary, and considered the monodromy operator there.
} 
that is, the singularities of the trace of $\cos \left(t \sqrt{-\Delta_{g}}\right)$ at $k T_{\gamma}$ come from a small neighbourhood of $\gamma$ (depending on $k$ ). As stated above we only need tangential pseudo-differential operators to localize near $\gamma$.

A straightforward translation to the semi-classical setting (see for instance [24]) gives (5.1). In fact, by standard oscillatory testing, $A\left(x,\left\langle D_{t}\right\rangle^{-1} D_{x}\right) e^{i t / h}=$ $e^{i t / h} \widetilde{A}\left(x, h D_{x} ; h\right)$, where $\widetilde{A}$ has the semi-classical principal symbol given by $A(x, \xi)$. This shows that for $\hat{f} \in \mathcal{C}_{\mathrm{c}}^{\infty}(\mathbb{R})$ even,

$$
\begin{aligned}
\operatorname{tr} f\left(\left(h \sqrt{-\Delta_{g}}-1\right) / h\right) & \tilde{A}\left(x, h D_{x}, h\right) \\
= & \frac{1}{2 \pi} \int \operatorname{tr}\left(A\left(x,\left\langle D_{t}\right\rangle^{-1} D_{x}\right) \cos t \sqrt{-\Delta_{g}}\right) \hat{f}(t) e^{i t / h} d t,
\end{aligned}
$$

and thus (5.2) follows from (5.1).

By combining Theorem 4, or rather Corollary 5.3, with Proposition 4.2 or with the geometric arguments of $[8, \S 2]$ we obtain the Weinstein conjecture first proved by Guillemin [8] in the case of elliptic trajectories, and by Zelditch $[20],[21]$ in general. It involves the concept of the Birkhoff normal form for the Hamiltonian - [8, Theorem 1.1]. As shown by Françoise and Guillemin [5] and Guillemin $[8, \S 2]$, and as can be also deduced from Section 4 above, that normal form is essentially equivalent to the normal form of the Poincaré map.

Corollary 5.4. Let $(M, g)$ be a compact Riemannian manifold without boundary, and $\gamma$ a simple non-degenerate geodesic on $M$, with the spectrum of its linear Poincaré map satisfying (2.9). Then the spectrum of the Laplacian, $\Delta_{g}$, determines the Birkhoff normal form of $\gamma$.

In the special case of symmetric domains in the plane, we can apply Theorem 5 and an observation of Colin de Verdière $[3, \S 4]$ to recover a result of Zelditch $[22]^{3}$

Corollary 5.5. Suppose that $\Omega \subset \mathbb{R}^{2}$ is a bounded domain, $\partial \Omega$ is real analytic, and that $\Omega$ is symmetric with respect to the $x$ and $y$ axes. Suppose also that $\Omega$ has a simple non-degenerate bouncing ball orbit coinciding with one of the axes, with multiplicity one in the length spectrum. Then the spectrum of the Dirichlet (or Neumann) Laplacian determines $\Omega$ among all such domains.

\section{Appendix}

In this appendix we outline an explicit construction of the quantum monodromy operator in a concrete geometric situation. ${ }^{4}$

\footnotetext{
${ }^{3}$ Melrose [15] has announced the same result, sketching a yet different proof. Recently, by considering the full quantum information, Zelditch [23] succeeded in removing one of the symmetry conditions.

${ }^{4}$ After this paper was written E. Heller and S. Tomsovic kindly pointed out to us that natural quantizations of Poincaré maps have been present in the physics literature since the work of Bogomolny [2].
} 
We reiterate that the abstract approach, recalled in Section 2 from [18], is all that is needed for our results and, in fact, we consider the minimal computational complexity the strength of our method.

Let $(X, g)$ be a compact $\mathcal{C}^{\infty}$ Riemannian $(n+1)$-manifold. We consider

$$
P=-h^{2} \Delta_{g}-1,
$$

where $\Delta_{g}$ is the Laplacian of the metric $g$. Suppose that $g$ has a closed geodesic, $\gamma_{0} \subset X$, and fix a point $x^{0} \in \gamma_{0}$. The geodesic lifts to a closed orbit, $\gamma$, of the Hamiliton flow of $p, p(x, \xi)=|\xi|_{g}^{2}-1$ on $p^{-1}(0)=S_{g}^{*} X \subset T^{*} X$. Let $m_{0}$ to be the lift of $x^{0}$. Let $Y$ be a $n$-submanifold of $X$ orthogonal to $\gamma_{0}$ at $x^{0}$, locally defined near $x^{0}$. Note that there are two possible lifts and we fix one of them.

The local kernel of $P$ near $m_{0}$ is defined as follows:

$u(h) \in \operatorname{ker}_{m_{0}}(P) \Longleftrightarrow W F_{h}(u(h)) \subset \operatorname{neigh}\left(m_{0}\right), \operatorname{neigh}\left(m_{0}\right) \subset \complement W F_{h}(P u(h))$,

where for

$$
u \in \mathcal{C}^{\infty}\left((0,1]_{h} ; \mathcal{D}^{\prime}(X)\right), \quad \exists N_{0}, \quad h^{-N_{0}} u \text { is bounded in } \mathcal{D}^{\prime}(X),
$$

we define

$$
\begin{aligned}
W F_{h}(u)=\complement\{(x, \xi): & \exists A \in \Psi_{h}^{0,0}(X) \sigma_{h}(A)(x, \xi) \neq 0, \\
& \left.A u \in h^{\infty} \mathcal{C}^{\infty}\left((0,1]_{h} ; \mathcal{C}^{\infty}(X)\right)\right\} .
\end{aligned}
$$

To avoid working with germs, we assume that the neighbourhoods in the definitions above are fixed.

We choose coordinates, $y \in \mathbb{R}^{n}$, on $Y$, so that $x^{0}$ corresponds to $y=0$. We can now identify $\operatorname{ker}_{m_{0}}(P)$ with $v(h) \in \mathcal{D}^{\prime}(Y), W F_{h}(v) \subset$ neigh $((0,0))$ using the solution operator:

$$
K: \mathcal{D}^{\prime}(Y) \longrightarrow \mathcal{D}^{\prime}(X), K v \in \operatorname{ker}_{m_{0}}(P),\left.\quad K v\right|_{Y}=v,
$$

which is microlocally defined near $\left((0,0), m_{0}\right)$ (for the precise formulation of microlocal definitions see the discussion preceding [18, Lemma 3.4]). To construct $K$ we choose, as local coordinates near $x^{0},(t, y)$, where $t=0$ on $Y$, and we can take it to be the distance along $\gamma_{0}$. In particular, $x^{0}$ corresponds to $(0,0)$, and $m_{0}$ to $((0,0),(1,0))$.

In these coordinates, the Schwartz kernel of $K$ is given by the usual expression (see for instance [11, Proposition 29.2] or [18, Proposition 7.3] for similar derivations):

$$
\begin{gathered}
K\left(t, y, y^{\prime}\right)=\frac{1}{(2 \pi h)^{n}} \int e^{\frac{i}{h}\left(\phi(t, y, \eta)-\left\langle y^{\prime}, \eta\right\rangle\right)} b(t, y, \eta ; h) d \eta \\
\left|d_{t, y} \phi\right|_{g}^{2}=1, \quad \phi(0, y, \eta)=\langle y, \eta\rangle, \quad d_{t, y} \phi(0,0,0)=(1,0), \\
\left(2 \nabla_{g} \phi \cdot \nabla_{g}+\Delta_{g} \phi\right) b=i h \Delta_{g} b, \quad b(0, y, \eta)=1 \quad \operatorname{near}(y, \eta)=(0,0),
\end{gathered}
$$

where the equation for $b$ is meant in terms of a formal power series in $h$, and $\nabla_{g}$ and $\cdot$ denote the Riemannian gradient and product, in the $(t, y)$ coordinates, respectively. 
The quantum flux norm on $\operatorname{ker}_{m_{0}}(P)$, thus microlocally identified with $\mathcal{D}^{\prime}(Y)$, is independent of $\chi$ which in this situation can be a function of the base variables only. An application of Green's formula in a tubular neighbourhood of $\gamma$, bounded by $Y$ on one side, shows that

$$
\begin{gathered}
\left\langle K v_{1}, K v_{2}\right\rangle_{\mathrm{QF}}=\frac{h}{i} \int_{Y}\left(\partial_{\nu}\left(K v_{1}\right) \overline{K v_{2}}-K v_{1} \overline{\partial_{\nu}\left(K v_{2}\right)}\right) d \operatorname{vol}_{Y}, \\
W F_{h}\left(v_{j}\right) \subset \operatorname{neigh}((0,0)), v_{j} \in \mathcal{D}^{\prime}(Y) \simeq \operatorname{ker}_{m_{0}}(P),
\end{gathered}
$$

where $\partial_{\nu}$ is the Riemannian normal derivative for $Y$ coinciding with $\partial_{t}$ at $(0,0)$, and $d \mathrm{vol}_{Y}$ is the volume form induced on $Y$ by $g$. Using (A.4) and (A.5) one can check that for $v$ with $W F_{h}(v) \subset \operatorname{neigh}((0,0))$,

$$
\|K v\|_{\mathrm{QF}} \sim\|v\|_{L^{2}(Y)} .
$$

We also see the relation with the traditional definition of the quantum flux, $\vec{J}$, normally presented in the context of Schrödinger equations:

$$
\vec{J}(u)=\frac{h}{i}\left(\nabla_{g} u \bar{u}-u \nabla_{g} \bar{u}\right), \quad u \in \operatorname{ker}_{m_{0}}(P) .
$$

Hence, if $\nu_{Y}$ is the unit normal vectorfield of $Y$ then

$$
\|u\|_{\mathrm{QF}}^{2}=\int_{Y} \vec{J}(u) \cdot \nu_{Y} d \operatorname{vol}_{Y}, \quad u \in \operatorname{ker}_{m_{0}}(P)
$$

that is our quantum flux norm $i s$ the usual quantum flux across the hypersurface $Y$. Again, the equalities are valid modulo $\mathcal{O}\left(h^{\infty}\right)$ for $u=K v$, with $W F_{h}(v)$ near $(0,0)$.

The simplest setting for writing down an expression for the quantum monodromy operator is given by $\gamma_{0}$ without conjugate points. In terms of its lift, $\gamma$, that condition is equivalent to demanding that for any $T>0$ there exists $U_{T}$, a neighbourhood of $m_{0}$ in $\left(S_{g}^{*}\right)_{x^{0}} X$, such that the projection

$$
\pi: \bigcup_{|t|<T} \exp \left(t H_{p}\right)\left(U_{T}\right) \longrightarrow X
$$

has surjective differentials.

Let $T_{0}$ be the primitive period of $\gamma_{0}$. To avoid focal points due to the curvature of $Y$, we now make one of many possible choices and put

$$
Y=\exp \left(2 T_{0} H_{p}\right)(U), \quad U \subset U_{2 T_{0}}=\operatorname{neigh}\left(m_{0}\right) \subset\left(S_{g}^{*}\right)_{x^{0}} X .
$$

We can identify a tubular neighbourhood of $\gamma_{0}$ with $Y \times \mathbb{S}_{T_{0}}^{1}$, where the second factor is the circle of length $T_{0}$. The absence of conjugate points, as reformulated above, shows that in a neighbourhood of $\gamma_{0}$ we can find a multivalued function $\phi=\phi(t, y, \eta), \eta \in \operatorname{neigh}(0)$, a parameter, such that

$$
\left|d_{t, y} \phi\right|_{g}^{2}=1, \quad \phi(0, y, \eta)=\langle y, \eta\rangle, \quad d_{t, y} \phi(0,0,0)=(1,0), \quad|t|<T .
$$

We can also solve for $b(t, y, \eta)$ in (A.4). 
If we take $T>T_{0}$ then we can use the phase and amplitude to write down the oscillatory integral representation of the quantum monodromy operator:

$$
\begin{gathered}
\mathcal{M}: \operatorname{ker}_{m_{0}}(P) \simeq \mathcal{D}^{\prime}(Y) \longrightarrow \mathcal{D}^{\prime}(Y) \simeq \operatorname{ker}_{m_{0}}(P), \\
v \longmapsto \mathcal{M} v(y)=\frac{1}{(2 \pi h)^{n}} \iint e^{\frac{i}{h}\left(\phi\left(T_{0}, y, \eta\right)-\left\langle y^{\prime}, \eta\right\rangle\right)} b\left(T_{0}, y, \eta ; h\right) v\left(y^{\prime}\right) d y^{\prime} d \eta \\
\|\mathcal{M} K v\|_{\mathrm{QF}}^{2}=\|K v\|_{\mathrm{QF}}^{2},
\end{gathered}
$$

with all the equalities valid microlocally near $(0,0)$, in particular, modulo $\mathcal{O}\left(h^{\infty}\right)$. The monodromy operator, $M$, can then be defined on $\mathcal{D}^{\prime}(Y)$, by $K M v=\mathcal{M} K v$.

The same construction is obviously valid for $P-z,|z| \leq 1-\delta, \delta>0$ and a detailed analysis of the solutions of the eikonal and transport equations could give a more precise description of $\mathcal{M}(z)$ in geometric terms. However, we can avoid that by using the Birkhoff normal forms of [12] and [13] for $\mathcal{M}(z)$ directly.

\section{Acknowledgements}

The third author would like to thank the National Science Foundation for partial support under grant DMS-9970614. He would also like to thank Bill Miller and Howard Taylor for references to the chemistry literature.

\section{References}

[1] N.W. Ashcroft, N.D. Mermin, Solid State Physics Saunders College Publishing, Philadelphia, 1976.

[2] E. Bogomolny, Semiclassical quantization of multi-dimensional systems, Nonlinearity 5 (1992), no. 4, 805-866.

[3] Y. Colin de Verdière, Sur les longeur de trajectoires périodic d'un billiard, South Rhone seminar on geometry, III (Lyon, 1983), 122-139, Travaux en Cours, Hermann, Paris, 1984.

[4] M. Dimassi, J. Sjöstrand, Spectral Asymptotics in the semi-classical limit, London Mathematical Society Lecture Note Series, 268. Cambridge University Press, Cambridge, 1999.

[5] J. P. Françoise, V. Guillemin, On the period spectrum of a symplectic mapping, J. Funct. Anal. 100 (1991), no. 2, 317-358.

[6] D. Fried, Cyclic resultants of reciprocal polynomials, Holomorphic dynamics (Mexico, 1986), 124-128, Lecture Notes in Math., 1345, Springer, Berlin, 1988.

[7] V. Guillemin, Wave trace invariants and a theorem of Zelditch, Internat. Math. Res. Notices 1993, no. 12, 303-308.

[8] — Wave trace invariants, Duke Math. J. 83 (1996), no. 2, 287-352.

[9] V. Guillemin, R. B. Melrose, The Poisson summation formula for manifolds with boundary, Adv. in Math. 32 (1979), no. 3, 204-232.

[10] B. Helffer, J. Sjöstrand, On diamagnetism and de Haas-van Alphen effect, Ann. Inst. H. Poincaré Phys. Théor. 52 (1990), no. 4, 303-375.

[11] L. Hörmander, The analysis of linear partial differential operators. III, IV, Grundlehren der Mathematischen Wissenschaften, 274, 275. Springer-Verlag, Berlin, 1985.

[12] A. Iantchenko, La forme normale de Birkhoff pour an opérateur intégral de Fourier, Asymptotic Anal. 17 (1998), no. 1, 71-92.

[13] A. Iantchenko, J. Sjöstrand, Birkhoff normal forms for Fourier integral operators. II, to appear in Amer. J. Math. Posted at http://xxx.lanl.gov/abs/math.SP/0111134

[14] M. P. Jacobson, C. Jung, H. S. Taylor, R. W. Field, State-by-state assignment of the bending spectrum of acetylene at $15000 \mathrm{~cm}^{-1}$ : A case study of quantum-classical correspondence, J. Chem. Phys. 111 (1999), no. 2, 600-618. 
[15] R. B. Melrose, The inverse spectral problem for planar domains (joint work with $V$. $W$. Guillemin), a talk at Berkeley, September, 1998.

[16] V. Petkov, L. Stoyanov, Geometry of reflected rays and inverse spectral problems, Pure and Applied Mathematics. John Wiley \& Sons, Ltd., Chichester, 1992.

[17] J. Sjöstrand, Semi-excited states in non-degenerate potential wells, Asymptotic Anal. 6 (1992), no. 1, 29-43.

[18] J. Sjöstrand, M. Zworski, Quantum monodromy and semi-classical trace formula, J. Math. Pure Appl. 81 (2002), 1-33.

[19] R. B. Shirts, W.P. Reinhardt, Approximate constants of motion for classically vibrational dynamics: Vague tori, semiclassical quantization, and classical intromolcular energy flow, J. Chem. Phys. 77 (1982), no. 10, 5204-5216.

[20] S. Zelditch, Wave invariants at elliptic closed geodesics, Geom. Funct. Anal. 7 (1997), no. 1, 145-213.

[21] Wave invariants for non-degenerate closed geodesics, Geom. Funct. Anal. 8 (1998), no. 1, 179-217.

[22] _ Spectral determination of analytic bi-axisymmetric plane domains, Geom. Funct. Anal. 10 (2000), no. 3, 628-677.

[23] The inverse spectral problem for analytic plane domains. I: Balian-Bloch trace formula, II: domains with symmetry, preprints, 2001:

http://arXiv.org/abs/math.SP/0111077, http://arXiv.org/abs/math.SP/0111078

[24] M. Zworski, A remark on a paper by E. B. Davies, Proc. Amer. Math. Soc. 129 (2001), no. $10,2955-2957$.

Malmö Högskola, Teknik och Samhälle, SE-20506, Malmö, Sweden.

E-mail address: ai@ts.mah.se

Centre de Mathématiques, École Polytechnique, FR-91128, Palaiseau Cedex, France, AND UMR 7640 OF CNRS.

E-mail address: johannes@math.polytechnique.fr

Department of Mathematics, University of California Berkeley, CA 94720, U.S.A.

E-mail address: zworski@math.berkeley.edu 